\title{
Polarisation basis transformation of weather radar measurements in the power domain
}

\author{
T. Otto ${ }^{1}$, J. Lu ${ }^{2}$, and M. Chandra ${ }^{3}$ \\ ${ }^{1}$ Atmospheric Remote Sensing, IRCTR, Delft University of Technology, The Netherlands \\ ${ }^{2}$ Former student at the Chair of Microwave Engineering and Photonics, Chemnitz University of Technology, Germany \\ ${ }^{3}$ Chair of Microwave Engineering and Photonics, Chemnitz University of Technology, Germany
}

\begin{abstract}
Polarisation diversity in radar remote sensing proved to be very successful in a variety of applications. Hydrometeors as raindrops or ice crystals are anisotropic radar targets giving rise to the use of polarisation diversity in weather radars. One advanced polarimetric weather radar is DLR's POLDIRAD in Oberpfaffenhofen. The huge data archive of this radar consists mainly of power measurements at diverse polarisation bases.

This study investigates the possibility to apply the polarisation basis transformation directly on power measurements. As a result, empirical transfer functions for the change of the polarisation basis of radar reflectivities are derived. To check their validity they are applied to appropriate polarimetric radar data from the POLDIRAD.
\end{abstract}

\section{Introduction}

Since 60 years weather radars have proven to be successful in the measurement of the rain rate over large areas. The first generation of weather radars measured only the power of the backscattered signal, later upgraded towards coherent systems to measure also the radial Doppler velocity.

Around 30 years ago, McCormick and Hendry (1975) started extensive research on polarimetric weather radars at the National Research Council of Canada using circular polarisation basis at S- and Ka-band frequencies.

Inspired by Seliga and Bringi (1976, 1978), the community started in the 1980s to investigate polarimetric weather radars transmitting alternately horizontal and vertical polarised pulses, receiving the backscattered echo in either of these polarisations or simultaneously in both.
A radar that combines all these approaches is the Polarisation Diversity Radar POLDIRAD of the German Aerospace Center (DLR) in Oberpfaffenhofen built in the mid 1980s. It uses a polarisation network based on ferrite phase shifters to transmit and receive arbitrarily linear, circular or elliptical polarisations. The backscattered echo is measured simultaneously by linear and logarithmic receivers. A technical description of this C-band $(5.45 \mathrm{~cm}$ wavelength) radar and its measurement principle is given in Schroth et al. (1988).

The data archive of the first 10 years of POLDIRAD's polarimetric measurements consists due to storage limitations mainly of power measurements, namely reflectivity measurements. An interesting set of data was taken under the aegis of the PADRE project, Chandra et al. (1994). During this project, stratiform storms were measured within a time interval lasting a few minutes at different polarisation bases. Using these data we investigate the possibility to perform polarisation basis transformations directly on reflectivity measurements.

The theory of the change of polarisation basis by means of a unitary basis transformation is well understood, Mott (2007). The polarisation basis transformation can be applied to coherent radar measurements if the full polarisation scattering matrix is measured, Schroth et al. (1988).

The approach we choose to transform directly the reflectivities is based on scattering simulations of rain at C-band. Empirical transfer functions between reflectivities at different polarisations are derived. To test their performance, these relationships are applied to the aforementioned radar data. 


\section{Basics}

\subsection{Polarisation of electromagnetic waves}

The free-space solution of the Helmholtz equation for the electric field of plane TEM waves yields

$$
\boldsymbol{E}(z, t)=\boldsymbol{E}_{\hat{\boldsymbol{x}}, \hat{\boldsymbol{y}}} \cdot \mathrm{e}^{-j k_{0} z} \cdot \mathrm{e}^{j \omega t}
$$

where $z$ is the propagation axis, $j=\sqrt{-1}, \omega$ is the angular frequency, $t$ is the time and $k_{0}$ is the free-space propagation constant. $\boldsymbol{E}_{\hat{\boldsymbol{x}}, \hat{\boldsymbol{y}}}$ the complex vector amplitude of the electric field also called Jones vector is given by

$$
\boldsymbol{E}_{\hat{\boldsymbol{x}}, \hat{\boldsymbol{y}}}=\left(\begin{array}{c}
E_{x} \\
E_{y}
\end{array}\right)=\left(\begin{array}{c}
E_{0 x} \mathrm{e}^{j \delta_{x}} \\
E_{0 y} \mathrm{e}^{j \delta_{y}}
\end{array}\right)
$$

where $\hat{\boldsymbol{x}}, \hat{\boldsymbol{y}}$ are orthonormal vectors forming the polarisation basis. Polarisation bases used for this work are linear verti$\mathrm{cal} /$ horizontal, linear slant and circular.

The backscattering properties of one single raindrop are described in terms of the backward scattering amplitude matrix at linear vertical/horizontal polarisation basis as defined by McCormick and Hendry (1975)

$$
\left(\begin{array}{c}
E_{v} \\
E_{h}
\end{array}\right)_{\hat{\boldsymbol{v}}, \hat{\boldsymbol{h}}}^{s}=\left(\begin{array}{ll}
S_{v v} & S_{v h} \\
S_{h v} & S_{h h}
\end{array}\right)_{\hat{\boldsymbol{v}}, \hat{\boldsymbol{h}}}\left(\begin{array}{c}
E_{v} \\
E_{h}
\end{array}\right)_{\hat{\boldsymbol{v}}, \hat{\boldsymbol{h}}}^{i} \cdot \frac{\mathrm{e}^{-j k_{0} R}}{R}
$$

where the superscripts ${ }^{i}$ and ${ }^{s}$ denote the incident wave at the scatterer and the scattered wave at the distance $R$ respectively. Raindrops are assumed to be oblate, symmetric, Pruppacher and Pitter (1971), and uncanted scatterers, Beard and Jameson (1983), resulting in the simplification of $S_{h v}=S_{v h}=0$. Under these assumptions linear vertical and horizontal polarisations are the principal polarisations which propagate through rain without being depolarised.

The polarisation basis transformation of the backward scattering amplitude matrix is given by

$$
\mathbf{S}_{\hat{e}_{1}, \hat{e}_{2}}=\mathbf{U}_{\hat{e}_{1}, \hat{e}_{2} \rightarrow \hat{v}, \hat{h}}^{\mathrm{T}} \mathbf{S}_{\hat{v}, \hat{h}} \mathbf{U}_{\hat{\boldsymbol{e}}_{1}, \hat{e}_{2} \rightarrow \hat{v}, \hat{h}}
$$

where the superscript ${ }^{T}$ stands for transposed, Mott (2007). The basis transformation matrix $\mathbf{U}_{\hat{\boldsymbol{e}}_{1}, \hat{\boldsymbol{e}}_{2} \rightarrow \hat{v}, \hat{\boldsymbol{h}}}$ is a $2 \times 2$ unitary matrix. The two columns of the basis transformation matrix are the Jones Vectors of the polarisations $\hat{\boldsymbol{e}}_{\mathbf{1}}$ and $\hat{\boldsymbol{e}}_{\mathbf{2}}$ expressed in the polarisation basis $\hat{\boldsymbol{v}}, \hat{\boldsymbol{h}}$.

The basis transformation matrix between vertical/horizontal $(\hat{\boldsymbol{v}}, \hat{\boldsymbol{h}})$ and left-/right-handed circular polarisations $(\hat{\boldsymbol{l}}, \hat{\boldsymbol{r}})$ and $+45^{\circ} /-45^{\circ}$ linear slant polarisations $(\hat{\jmath}, \hat{\jmath})$ are

$$
\mathbf{U}_{\hat{\imath}, \hat{r} \rightarrow \hat{\boldsymbol{v}}, \hat{\boldsymbol{h}}}=\frac{1}{\sqrt{2}}\left(\begin{array}{rr}
j & -j \\
1 & 1
\end{array}\right)
$$

and

$$
\mathbf{U}_{\hat{\jmath}, \hat{\imath} \rightarrow \hat{\boldsymbol{v}}, \hat{\boldsymbol{h}}}=\frac{1}{\sqrt{2}}\left(\begin{array}{rr}
1 & 1 \\
1 & -1
\end{array}\right)
$$

resulting in the transformed backward scattering amplitude matrices

$$
\mathbf{S}_{\hat{l}, \hat{r}}=\left(\begin{array}{ll}
S_{l l} & S_{l r} \\
S_{r l} & S_{r r}
\end{array}\right)=\frac{1}{2}\left(\begin{array}{ll}
S_{h h}-S_{v v} & S_{h h}+S_{v v} \\
S_{h h}+S_{v v} & S_{h h}-S_{v v}
\end{array}\right)
$$

and

$$
\mathbf{S}_{\hat{\jmath}, \hat{\imath}}=\left(\begin{array}{l}
S_{/ /} S_{\bigwedge} \\
S_{\backslash /} S_{\backslash \backslash}
\end{array}\right)=\frac{1}{2}\left(\begin{array}{l}
S_{h h}+S_{v v} S_{v v}-S_{h h} \\
S_{v v}-S_{h h} S_{h h}+S_{v v}
\end{array}\right) .
$$

It should be pointed out that at linear vertical/horizontal polarisation basis the anisotropy of the oblate raindrops shows up in the main diagonal of the scattering matrix. However, at circular or linear slant polarisation basis this information is contained within the columns of the scattering matrix.

\subsection{Scattering simulation of rain}

To simulate the backward scattering amplitudes of raindrops, the Fredholm integral method (FIM) for dielectric spheroids was used, Holt et al. (1978). All raindrops were assumed to be equally aligned with their major axis along horizontal polarisation without any canting. The empirical relationships of Ray (1972) were applied to calculate the dielectric properties of the raindrops.

Three raindrop shape models served as input to the scattering simulation

- Pruppacher and Beard (1970),

- Keenan et al. (2001),

- and a combination of Keenan et al. (2001), Andsager et al. (1999) and Beard and Chuang (1987).

A gamma drop size distribution normalised with respect to the liquid water content, Bringi and Chandrasekar (2001), was used, given as

$$
N(D)=N_{w} f(\mu)\left(\frac{D}{D_{0}}\right)^{\mu} \mathrm{e}^{-(3.67+\mu) \frac{D}{D_{0}}}
$$

with

$$
f(\mu)=\frac{6}{3.67^{4}} \frac{(3.67+\mu)^{\mu+4}}{\Gamma(\mu+4)}
$$

where $\Gamma$ is the gamma function.

With the backward scattering amplitudes for horizontal $S_{h h}$ and vertical polarisations $S_{v v}$, obtained by the scattering simulations, the polarisation basis transformations according to Eqs. (7) and (8) were performed. Using the radar cross section given as

$$
\sigma_{x y}=4 \pi \cdot\left|S_{x y}\right|^{2}
$$

the reflectivities $z_{x y}$ were calculated as

$$
z_{x y}=\frac{\lambda^{4}}{\pi^{5}|K|^{2}} \cdot \int_{D} \sigma_{x y}(D) N(D) \mathrm{d} D
$$


where $\lambda$ is the wavelength in $\mathrm{mm},|K|^{2}$ is a dielectric factor and $\sigma_{x y}$ is the radar cross section in $\mathrm{mm}^{2}$ for an incident wave of polarisation $y$ and a scattered wave of polarisation $x$. The integration was performed for raindrops with an equivolumetric diameter $D$ ranging from $0.1 \mathrm{~mm}$ to $8 \mathrm{~mm}$. Propagation effects such as attenuation and differential propagation phase were neglected.

Usually the reflectivity is given in logarithmic units as

$$
Z_{x y}=10 \cdot \log _{10}\left(\frac{z_{x y}}{\mathrm{~mm}^{6} \mathrm{~m}^{-3}}\right)(\mathrm{dBZ}) .
$$

To model the natural variations of rainfall, 1500 simulations were performed. For each simulation the temperature was varied randomly between $1^{\circ} \mathrm{C}$ and $25^{\circ} \mathrm{C}$. Furthermore, one of the three axis ratios was randomly selected. The three parameters of the normalised gamma distribution were varied as $2 \leq \log N_{w} \leq 5,0.5 \mathrm{~mm} \leq D_{0} \leq 3.5 \mathrm{~mm}$ and $-1 \leq \mu \leq 5$. The set of input parameters was chosen in a way that the resulting distribution of the rain rate satisfies with close agreement the disdrometer measurements from Hagen (2001).

The reflectivities are determined by the weather radar measuring the backscattered and received power simultaneously in the co- and the cross-polarised receiver channels by logarithmic receivers. The reflectivity is then calculated using the radar equation for distributed targets as

$$
Z_{x y}=10 \cdot \log _{10}\left(C \cdot R^{2} \cdot \bar{P}_{x y}\right)(\mathrm{dBZ})
$$

with the radar constant $C, R$ the range between the radar and the radar pulse volume and $\bar{P}_{x y}$ the mean received power.

\subsection{Radar data}

Figure 1 shows an example set of reflectivity measurements taken with the POLDIRAD. Shown are vertical cuts (rangeheight indicators, RHI's) of stratiform summer storms. As mentioned, the uniqueness of these datasets is that the storm was measured within a time interval last a few minutes at different polarisation bases. In all datasets the storm was first measured in linear vertical/horizontal polarisation basis, followed few minutes later by a measurement in linear slant polarisation basis and again few minutes later in circular polarisation basis. The reflectivities $Z_{h h}$ and $Z_{\backslash \backslash}$ look qualitatively almost equal. $Z_{l l}$ is, as expected, much weaker, Eq. (7).

As this study only considers the polarisation basis transformation of reflectivities measured in rain, hence the whole datasets could not be used to validate the empirical reflectivity transfer functions derived in the next section. Only radar data from rain areas were used. From the RHI's of Fig. 1 a rectangular area in the range from $36 \mathrm{~km}$ until $50 \mathrm{~km}$, at heights of $0 \mathrm{~km}$ up to $2 \mathrm{~km}$ was manually selected. To minimise ground clutter echoes, the minimum elevation angle considered was $0.5^{\circ}$.

In all, radar data from four days were used, covering 2158 radar pulse volumes of rain only.
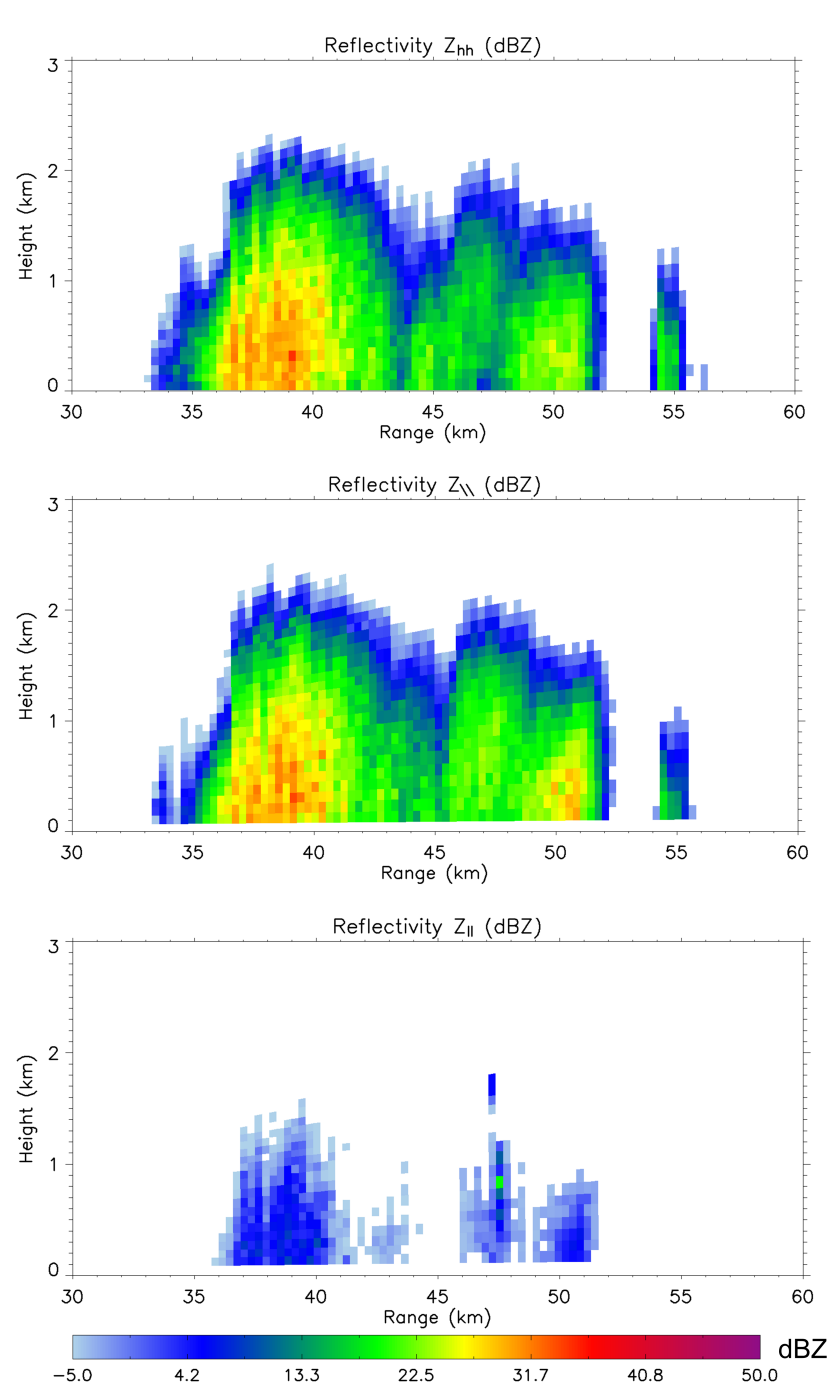

Fig. 1. Range-height indicators of the reflectivities $Z_{h h}, Z_{\backslash \backslash}$ and $Z_{l l}$ (dBZ) measured the 4 th of June 1993 at 11:50 a.m., 11:51 a.m. and 11:52 a.m. respectively.

\section{Empirical relations between reflectivities for polarisa- tion basis transformation}

Following the scattering simulation approach described in the preceding section, reflectivities at different polarisation bases were calculated and plotted against each other, black dots in Fig. 2.

As one can see from Eqs. (7), (8), (11), (12) and (13) $Z_{r l}=Z_{l r}=Z_{/ /}=Z_{\backslash \backslash}$ and $Z_{l l}=Z_{r r}=Z_{\backslash /}=Z_{\bigwedge}$ in the absence of propagation effects and for symmetric raindrops all equally aligned and uncanted. Therefore, two empirical relations are enough to transform $Z_{h h}$ into these reflectivities.

The two linear reflectivity transfer functions that were derived are

$$
Z_{/ /, \backslash \backslash, l r, r l}(\mathrm{dBZ})=0.98 \cdot Z_{h h}(\mathrm{dBZ})+0.25
$$




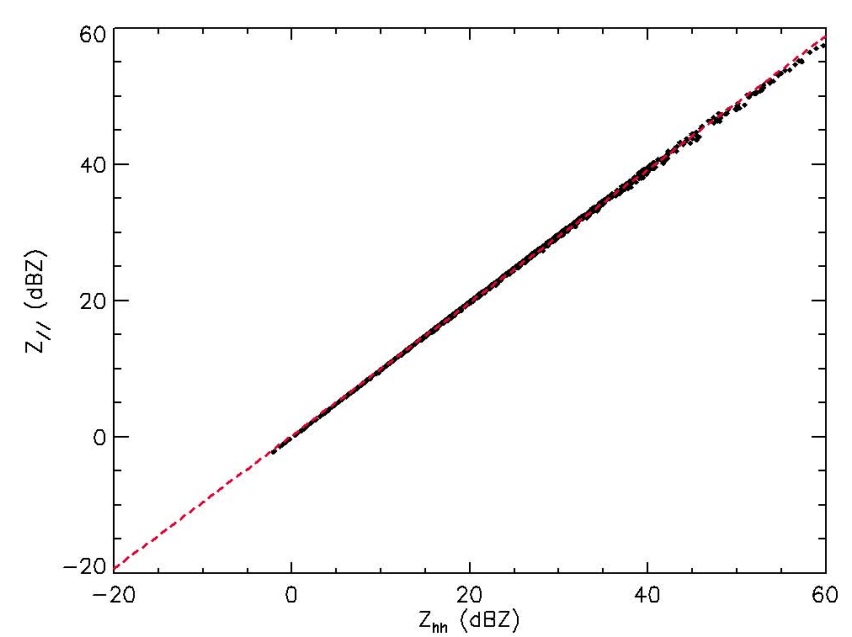

(a)

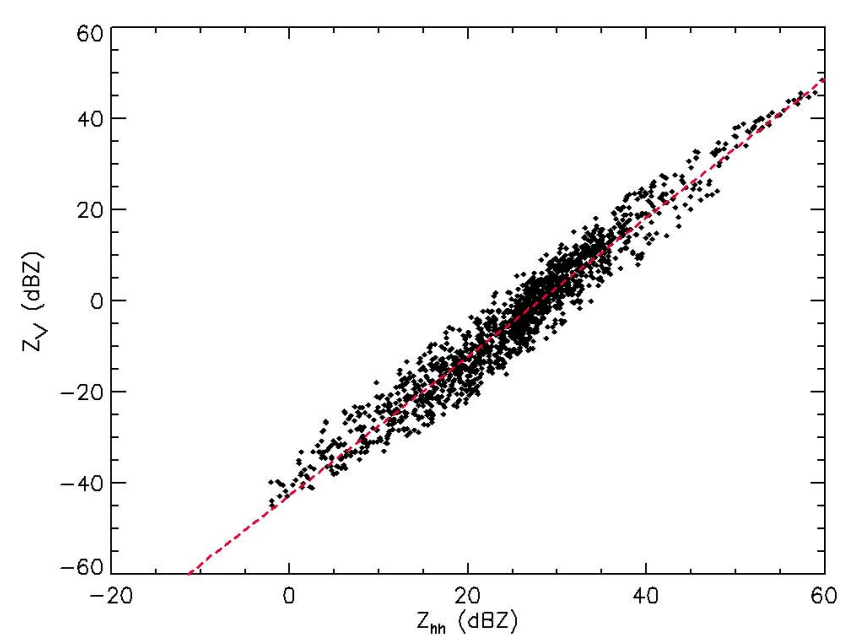

(b)

Fig. 2. Scattering simulation results for reflectivities (black) and their linear fits (red dashed lines), (a) $Z_{/ /}=\mathrm{f}\left(Z_{h h}\right)$, Eq. 15, (b) $Z_{\bigvee}=\mathrm{f}\left(Z_{h h}\right)$, Eq. 16.

and

$$
Z_{\backslash, \wedge, l l, r r}(\mathrm{dBZ})=1.52 \cdot Z_{h h}(\mathrm{dBZ})-42.66 .
$$

They are depicted as dashed red curves in Fig. 2 (a) and (b). The standard deviation of the fit for $Z_{/ /, \backslash \backslash, l r, r l}$ in comparison to the simulation results is $0.22 \mathrm{~dB}$ and for $Z \backslash /, \wedge, l l, r r$ the standard deviation is $3.99 \mathrm{~dB}$.

The large standard deviation for the $Z \backslash /, \wedge, l l, r r-Z_{h h}$ fit might be explained, expanding the radar cross sections as

$$
\sigma_{r l / l l}=\pi \cdot\left(\left|S_{h h}\right|^{2}+\left|S_{v v}\right|^{2} \pm 2\left|S_{h h}\right|\left|S_{v v}\right| \cos \delta\right)
$$

with

$$
\delta=\angle S_{h h}-\angle S_{v v}
$$

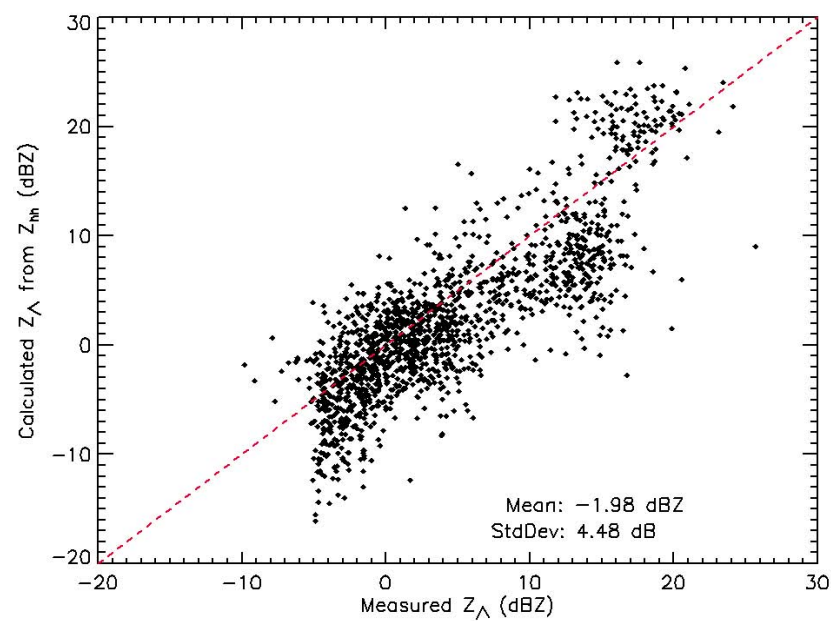

(a)

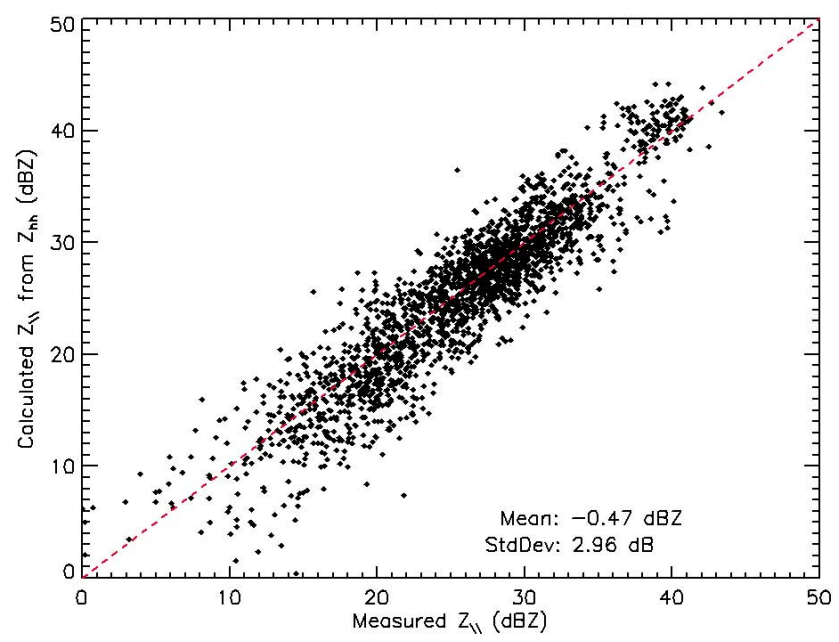

(b)

Fig. 3. Test of the reflectivity transfer functions with radar data for (a) $Z_{\bigwedge}$ and (b) $Z_{\backslash \backslash}$. Plotted along the $\mathrm{x}$-axis are the measured reflectivities $(\mathrm{dBZ})$ and plotted on the $y$-axis are the reflectivities at the same polarisation estimated from the measured reflectivity $Z_{h h}$ (dBZ) using the reflectivity transfer functions, Eqs. (15) and (16).

The difference between $\sigma_{r l}$ and $\sigma_{l l}$ is the sign before the last term in Eq. (17) that contains the differential phase on backscattering $\delta$. Especially the weak echo $\sigma_{l l}$ is sensitive to small changes of this phase.

Note that for $\delta>90^{\circ}$ the weak echo becomes the strong one and vice versa. The differential phase on backscattering of rain alone will not reach such high values at $\mathrm{C}$-band, but there might exist a differential system phase offset or the differential propagation phase that contributes to this term. 


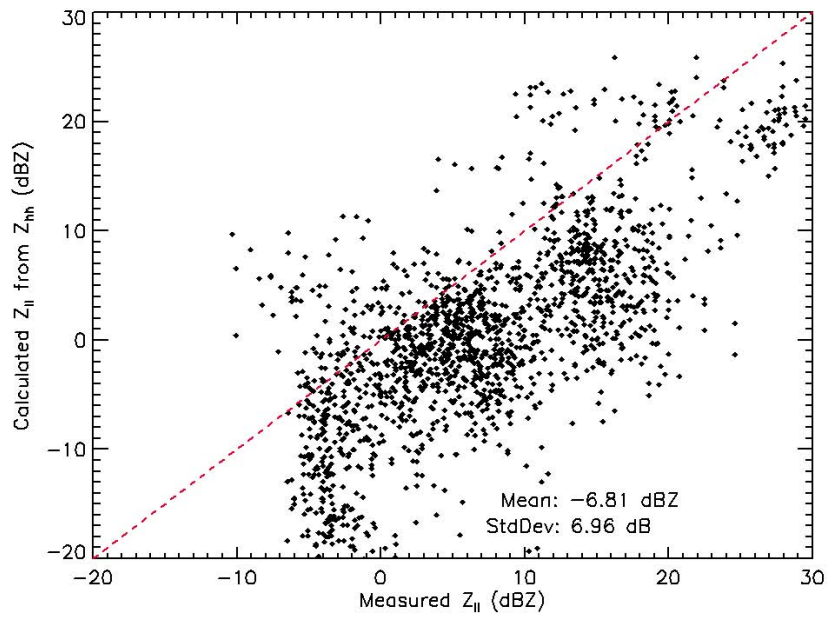

(a)

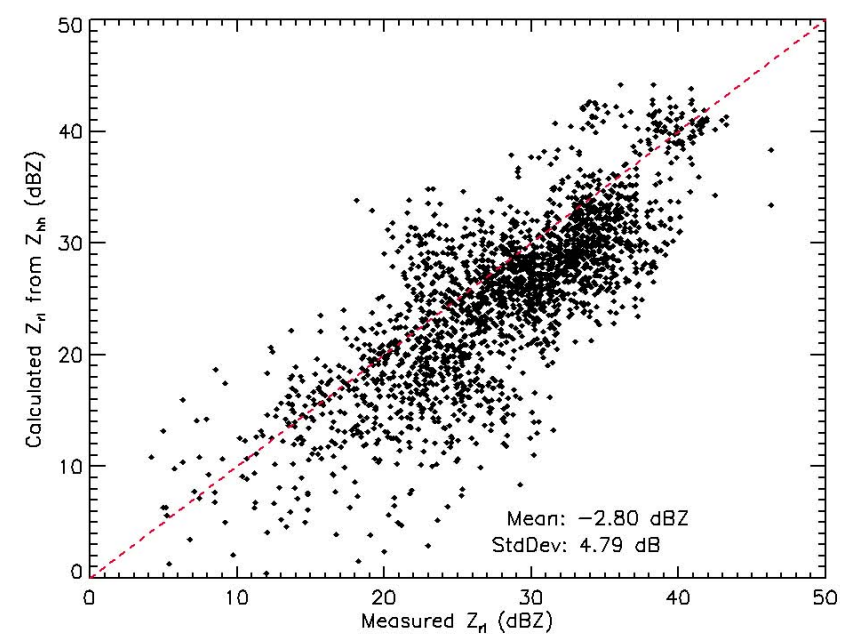

(b)

Fig. 4. Same as Fig. 3 but with radar data at circular polarisation basis, (a) $Z_{l l}$ and (b) $Z_{r l}$.

\section{Application to polarimetric radar data}

The empirical functions to transform the polarisation basis of reflectivities, Eqs. (15) and (16), were applied to the radar data presented in Sect. 2.3. The results are shown in form of scatter plots in Figs. 3 and 4. Plotted are the measured reflectivities in $\mathrm{dBZ}$ along the $\mathrm{x}$-axis and along the $\mathrm{y}$-axis the reflectivity at the same polarisation basis calculated from $Z_{h h}$ (dBZ) using the reflectivity transfer functions, Eqs. (15) and (16). Also shown in the figures are the mean offset and the standard deviation from the $y=x$ line (dashed red line).

The best result shows the co-polar reflectivity at linear slant polarisation basis $Z_{\backslash \backslash}$ with a mean deviation of -0.47 $\mathrm{dB}$ between the measurement and the application of the reflectivity transfer function to $Z_{h h}$, Eq. (15). The standard deviation of about $3 \mathrm{~dB}$ is probably mainly attributed to the time interval of one to three minutes between the measurements

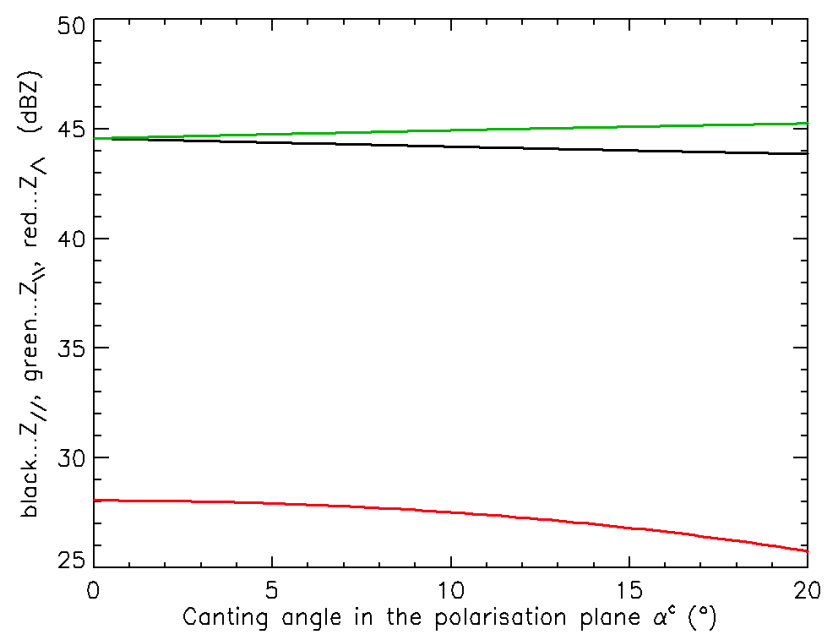

Fig. 5. Dependence of $Z_{/ /}, Z_{\backslash \backslash}$ and $Z_{/}$on the effective canting angle in the polarisation plane for a rain rate of $20 \mathrm{~mm} / \mathrm{h}$.

of the storm in linear vertical/horizontal and linear slant polarisation basis. This time interval between the successive measurements is large enough for the measured reflectivities to contain random and systematic drifts. At any rate the time interval is significantly larger than the time scale of decorrelation, Tracksdorf et al. (2005). The higher deviations for the results at circular polarisation basis, Fig. 4, are also reasonable because the measurement at circular polarisation basis was always the last one.

There exists another effect that might be responsible for the deviations between linear slant and circular polarisation basis. That is, that the reflectivities at circular polarisation basis are insensitive to raindrop canting angle variations whereas the reflectivities at linear slant polarisation basis are not. To quantify this influence, the scattering simulation was expanded. A raindrop canting angle $\alpha$ in the polarisation plane, Holt (1984), was included. The dependence of the reflectivities $Z_{/ /}, Z_{\backslash \backslash}$ and $Z_{/}$on the raindrop canting angle $\alpha$ in the plane of polarisation is shown in Fig. 5. The simulation was performed for an exponential Marshall-Palmer drop size distribution, Marshall and Palmer (1948), and a rain rate of $20 \mathrm{~mm} / \mathrm{h}$.

It is expected that the raindrop canting angle is narrowly distributed with a mean close to zero, Beard and Jameson (1983). The simulation results indicate that for these small canting angles the variations of the reflectivities are well below the measurement accuracy of $1 \mathrm{~dB}$. So, it is concluded that canting angle effects will have a very marginal effect on the results of our polarisation basis transformation of the reflectivities.

The higher standard deviations for $Z_{八}$ and $Z_{l l}$ compared to the standard deviations for $Z_{\backslash \backslash}$ and $Z_{r l}$ are in agreement with the simulation results. We also note that the signal to noise ratios are less favourable in the weaker channels. 


\section{Conclusions}

Scattering simulations of rain at C-band were performed in order to obtain empirical functions to transform directly the polarisation basis of reflectivities. In all, two linear functions were presented to transform the reflectivity $Z_{h h}$ into the coand cross-polarised reflectivities at linear slant and circular polarisation basis.

These relations were applied successfully to polarimetric weather radar data. Better results were obtained for the transformation from $Z_{h h}$ into linear slant polarisation basis than into circular polarisation basis. The simulation showed that these differences can not be due to raindrop canting angle effects on backscattering. More likely responsible, amongst others, is the time lag between the measurements at different polarisation bases. The order in which the measurements took place was first at linear vertical / horizontal polarisation basis, followed by the measurement at linear slant polarisation basis and finally at circular polarisation basis. The time lag between the measurements was always in the order of one to three minutes, long enough to cause a drift in the meteorological state. A more meaningful check of the reflectivity transfer functions might be achieved when the storm is measured at different polarisation basis in time intervals in the order of seconds.

The backscattering simulation revealed that the weak echo component at linear slant and circular polarisation basis is prone to even small changes of the differential phase on backscattering. In weather radar measurements, this phase will be superimposed by the differential propagation phase. Therefore, further work has to include the modeling of propagation effects that deteriorate the polarisation of the transmitted linear slant and circular polarised wave.

Acknowledgements. The authors thank the late Anthony R. Holt of the University of Essex, UK, for providing the Fredholm Integral Method (FIM) scattering code for dielectric spheroids and Martin Hagen, DLR Germany, for providing the disdrometer rain rate statistics.

\section{References}

Andsager, K., Beard, K. V., and Laird, N. F.: Laboratory Measurements of Axis Ratios for Large Raindrops, J. Atmos. Sci., 56, 2673-2683, 1999.

Beard, K. V. and Chuang, C.: A New Model for the Equilibrium Shape of Raindrops, J. Atmos. Sci., 44, 1509 - 1524, 1987.

Beard, K. V. and Jameson, A. R.: Raindrop Canting, J. Atmos. Sci., 40, 448-454, 1983.
Bringi, V. N. and Chandrasekar, V.: Polarimetric Doppler Weather Radar, Cambridge University Press, 1st edn., p. 410, 2001.

Chandra, M., Wood, S. J., Lux, S., Holt, A. R., and Schroth, A.: The PADRE Project, European Commission COST 75 Weather Radar Systems, edited by: Collier, C. G., in: Report EUR 16013 EN, ISSN 1018-5593, 507-518, Brussels, Belgium, 1994.

Hagen, M.: On the variation of the parametrization of rainfall rate estimation by dual polarization techniques, in: 30th Conference on Radar Meteorology, Preprints, 11B.3, Munich, Germany, 2001.

Holt, A. R.: Some factors affecting the remote sensing of rain by polarization diversity radar in the $3-$ to $35-\mathrm{GHz}$ frequency range, Radio Sci., 19, 1399-1412, 1984.

Holt, A. R., Uzunoglu, N. K., and Evans, B. G.: An Integral Equation Solution to the Scattering of Electromagnetic Radiation by Dielectric Spheroids and Ellipsoids, IEEE T. Antennas Propag., AP-26, 706-712, 1978.

Keenan, T. D., Carey, L. D., Zrnic, D. S., and May, P. T.: Sensitivity of 5-cm Wavelength Polarimetric Radar Variables to Raindrop Axial Ratio and Drop Size Distribution, J. Appl. Meteor., 40, 526-545, 2001.

Marshall, J. S. and Palmer, W. M.: The Distribution of Raindrops with Size, J. Meteorol., 5, 165-166, 1948.

McCormick, G. C. and Hendry, A.: Principles for the radar determination of the polarization properties of precipitation, Radio Sci., 10, 421-434, 1975.

Mott, H.: Remote Sensing with Polarimetric Radar, John Wiley \& Sons, 1st edn., 68-70, 2007.

Pruppacher, H. R. and Beard, K. V.: A wind tunnel investigation of the internal circulation and shape of water drops falling at terminal velocity in air, Q. J. Roy. Meteor. Soc., 96, 247-256, 1970.

Pruppacher, H. R. and Pitter, R. L.: A Semi-Empirical Determination of the Shape of Cloud and Rain Drops, J. Atmos. Sci., 28, 86-94, 1971.

Ray, P. S.: Broadband Complex Refractive Indices of Ice and Water, Appl. Optics, 11, 1836-1844, 1972.

Schroth, A. C., Chandra, M. S., and Meischner, P. F.: A C-Band coherent polarimetric radar for propagation and cloud physics research, J. Atmos. Oceanic Technol., 5, 803-822, 1988.

Tracksdorf, P., Ghorbani, A., Chandra, M., Hagen, M., and Bebbington, D.: Comparison of linear and logarithmic receiver signals from polarimetric weather radar echoes and their temporal decorrelation properties, Adv. Radio Sci., 3, 401-411, 2005 , http://www.adv-radio-sci.net/3/401/2005/. 